\title{
Rates of complex formation in collisions of rotationally excited homonuclear diatoms with ions at very low temperatures: Application to hydrogen isotopes and hydrogen-containing ions ${ }^{\text {a) }}$
}

\author{
E. I. Dashevskaya \\ Department of Chemistry, Technion-Israel Institute of Technology, Haifa 32000, Israel and \\ Max-Planck-Institut für Biophysikalische Chemie, Am Fassberg, D-37077 Göttingen, Germany \\ I. Litvin \\ Department of Chemistry, Technion-Israel Institute of Technology, Haifa 32000, Israel and Institut \\ für Physikalische Chemie der Universität Gottingen, Tammannstrasse 6, D-37077 Göttingen, Germany \\ E. E. Nikitin \\ Department of Chemistry, Technion-Israel Institute of Technology, Haifa 32000, Israel and \\ Max-Planck-Institut für Biophysikalische Chemie, Am Fassberg, D-37077 Göttingen, Germany \\ J. Troe \\ Max-Planck-Institut für Biophysikalische Chemie, Am Fassberg, D-37077 Göttingen, Germany and Institut \\ für Physikalische Chemie der Universität Gottingen, Tammannstrasse 6, D-37077 Göttingen, \\ Germany
}

(Received 14 December 2004; accepted 21 February 2005; published online 10 May 2005)

\begin{abstract}
State-selected rate coefficients for the capture of ground and rotationally excited homonuclear molecules by ions are calculated, for low temperatures, within the adiabatic channel classical $(\mathrm{ACCl})$ approximation, and, for zero temperature, via an approximate calculation of the Bethe limit. In the intermediate temperature range, the accurate quantal rate coefficients are calculated for $j$ $=0$ and $j=1$ states of hydrogen isotopes $\left(\mathrm{H}_{2}, \mathrm{HD}\right.$, and $\left.\mathrm{D}_{2}\right)$ colliding with hydrogen-containing ions, and simple analytical expressions are suggested to approximate the rate coefficients. For the ground rotational state of diatoms, the accurate quantal rate coefficients are higher compared to their $\mathrm{ACCl}$ counterparts, while for the first excited rotational state the reverse is true. The physical significance of quantum effects for low-temperature capture and the applicability of the statistical description of capture are considered. Particular emphasis is given to the role of Coriolis interaction. The relevance of the present capture calculations for rates of ortho-para conversion of $\mathrm{H}_{2}$ in collisions with hydrogen-containing ions at low temperatures is discussed. (C) 2005 American Institute of Physics. [DOI: $10.1063 / 1.1889425]$
\end{abstract}

\section{INTRODUCTION}

The rates of many barrierless exothermic reactions are determined by the rates of complexes formation in collisions of the reagents. The most simple method for calculating the rates of complex formation (or of capture), at not too high temperatures, is the adiabatic channel (AC) approach. ${ }^{1}$ It is based on the assumption of adiabatic relative motion of the colliding partners across central AC potentials. The latter are defined as eigenvalues of the matrix of the total potential of the interacting partners in representation in which the collision axis $\mathbf{R}$ is taken as quantization axis of the intrinsic angular momenta of the partners. Thus, the total projection of the intrinsic angular momenta $\omega$ is assumed to be an exact quantum number. If, in addition, the relative motion is treated classically this AC approach, which will be abbreviated by $\mathrm{ACCl}$ (adiabatic channel classical) becomes especially simple. It has been used for the calculation of statespecific rate constants of many practically important

\footnotetext{
a) Dedicated to Professor J. Wolfrum at the occasion of his 65th birthday.
}

processes. $^{2}$ The AC approach ignores nonadiabatic effects which arise from the relative radial and rotational motion of the partners. The implication of this approximation was discussed recently. ${ }^{3,4}$ It was argued that for ion-diatom collisions at very low temperatures (say, below $1 \mathrm{~K}$ ), the capture dynamics simplifies in two respects: the AC potentials can be calculated in the perturbed rotor approximation and the radial nonadiabatic coupling can be safely neglected. On the other hand, the relative motion of the partners should be treated quantum mechanically rather than classically.

In the present paper we report the results of calculations of capture rate coefficients for rotationally excited stateselected homonuclear diatomics by ions at temperatures $T$ which are substantially below the characteristic rotational temperature of a diatomic, $T_{\text {rot }}=B / k_{\mathrm{B}}$, where $B$ is the rotational constant of the molecule (in energy units). An interesting feature of these collisions is a long-range anisotropic charge-quadrupole interaction for rotationally excited molecules which would cause a divergence of the $\mathrm{ACCl}$ rate coefficients at the limit of zero temperature. This divergence is an artifact of the $\mathrm{ACCl}$ approximation, the range of valid- 
ity of which can be established by comparing $\mathrm{ACCl}$ rate coefficients with estimated correct quantum zero-temperature rate coefficients. The accurate bridging between $\mathrm{ACCl}$ rate coefficients and the correct zero-temperature limit is performed in the present work for collisions of hydrogen isotope diatomics $\left(\mathrm{H}_{2}, \mathrm{HD}, \mathrm{D}_{2}\right)$ in $j=0$ and $j=1$ states with diatomic and polyatomic hydrogen-containing ions. The choice of these systems was motivated by the fact that such processes play an important role in atmospheric and interstellar chemistry, for instance, in cascading down the rotational ladder of hydrogen in cold translational environment. ${ }^{5,6}$ It should also be noted also that collisions of hydrogen molecules are the best candidates for the manifestation of specific lowtemperature features of complex formation, and that hydrogen-containing ions are partners that provide the interesting opportunity to investigate effects originating from the identity of protons (the so-called exchange symmetry ${ }^{5,6}$ ) and relating to the intramolecular conversion of nuclear spins (nonconservation of the total spin of protons in the complex ${ }^{7}$ ).

This paper is arranged in the following way. In Sec. II we discuss different regimes of low-temperature capture and we comment on some general aspects of the statistical theory of capture. In Sec. III we report the results of ACCl capture calculations for arbitrary rotational quantum numbers $j$ of the diatom. In Sec. IV we consider the zero-temperature limit of the quantum capture rate for arbitrary $j$. In Sec. V we consider the qualitative behavior of capture rate constants $k_{j}$ across the very low-temperature range, present accurate results for $k_{j=0}$ and $k_{j=1}$ of hydrogen molecules, and also suggest simple analytical expressions for the rate coefficients that bridge the gap between the zero-temperature and the classical adiabatic channel limits. Section VI concludes the paper with a qualitative discussion of ortho-para conversion of hydrogen molecules in collisions with hydrogencontaining ions.

\section{DIFFERENT REGIMES OF LOW-TEMPERATURE CAPTURE}

The interaction energy between a homonuclear diatom and an ion at long range is determined by isotropic chargeinduced dipole and anisotropic charge-induced dipole plus charge-quadrupole terms. The interaction potential depends on the interfragment distance $R$ and the angle $\gamma$ between the molecular axis and the collision axis: ${ }^{8}$

$$
U(R, \gamma)=-\frac{q^{2} \alpha}{2 R^{4}}+\left(-\frac{q^{2} \Delta \alpha}{3 R^{4}}+\frac{q Q}{R^{3}}\right) P_{2}(\cos \gamma) .
$$

Here $\alpha=\left(\alpha_{\|}+2 \alpha_{\perp}\right) / 3$ is the mean polarizability, $\Delta \alpha=\alpha_{\|}$ $-\alpha_{\perp}$ denotes the polarizability anisotropy, $Q$ is the quadrupole moment of the molecule, $q$ is the charge of the ion, and $P_{2}$ is the Legendre polynomial. The product $q Q$ may be positive or negative (the so-called even case for $q Q>0$ and odd case for $\mathrm{qQ}<0$ ).

Considering the low-temperature (LT) region, $T \ll T_{\text {rot }}$ $=B / k_{\mathrm{B}}$, we note two features that simplify the capture dynamics: (i) the capture proceeds adiabatically with respect to rotational transitions and (ii) the full interaction potential can be simplified by making use of the perturbed rotor (PR) approximation.

The property (i) follows from the Massey parameter for rotational transitions $\xi=B \Delta R / \hbar \nu$ where $\nu$ is the collision velocity and $\Delta R$ is the range of the potential. Taking the latter as the Langevin capture radius $R_{L}=\left(q^{2} \alpha / 2 E\right)^{1 / 4}$, where $E$ is the collision energy, and estimating $\alpha$ as $\alpha \approx r_{0}^{3}$ with $r_{0}$ being the "complex radius," we write

$$
\xi=\frac{B \Delta R}{\hbar \nu} \sim \frac{B R_{L}}{\hbar \nu} \sim\left(\frac{B}{E}\right)^{3 / 4}\left(\frac{q^{2}}{r_{0} B}\right)^{3 / 4} .
$$

The first factor on the right-hand side (rhs) of Eq. (2) at $T$ $\ll T_{\text {rot }}$ obviously is larger than unity. The second factor is also larger than unity, since $\left(q^{2} / r_{0} B\right)$ represents the ratio of the Coulomb energy at the place of the radius of the complex to the rotational constant. The condition $\xi \gg 1$ means that there are no transitions between rotational states of the diatom on the way to complex formation.

Property (ii) follows from the comparison of the interaction energy $U$ at the capture distance $R_{L}$ with the rotational constant

$$
\frac{U\left(R_{\mathrm{L}}\right)}{B} \sim \frac{q^{2} \alpha}{R_{L}^{4} B} \sim \frac{E}{B} \ll 1 .
$$

The condition $U\left(R_{L}\right) / B \ll 1$ means that the expression for $U$ can be simplified by using the PR approximation. The PR interaction energy is calculated by using the collision axis $\mathbf{R}$ as the quantization axis for the intrinsic angular momentum $j$ which is assumed to correspond to a good quantum number. The projection quantum number $\omega$ serves, together with $j$, in specifying the adiabatic channel potentials $V_{\omega}^{j}(R)$ and the matrix of centrifugal energy $C_{\omega, \omega^{\prime}}^{J, j}(R)$. The diagonal elements of the latter are the centrifugal potential $C_{\omega, \omega}^{J, j}(R)$, while the off-diagonal elements $C_{\omega, \omega \pm 1}^{J, j}(R)$ correspond to the Coriolis interaction between states with different $\omega$. Within this approach, the capture is described by the solution of halfcollision quantum scattering equations, which in the total angular momentum representation $J j \omega$ can be decomposed into two blocks with dimensions of $(j+1)$ and $j$, differing in parity. The explicit expressions for the matrix elements, which enter into the scattering equations and which are generated by the potential in Eq. (1) and by the relative rotation, $\operatorname{are}^{8}$

$$
\begin{aligned}
V_{0}^{0}(R) & =\left.\langle J j \omega|U(R, \gamma)| J j \omega\rangle\right|_{j=0, \omega=0}=-\frac{q^{2} \alpha}{2 R^{4}}, \\
V_{\omega}^{j}(R) & =\left.\langle J j \omega|U(R, \gamma)| J j \omega\rangle\right|_{j>0} \\
& =-\frac{q^{2} \alpha}{2 R^{4}}+\frac{\left[j(j+1)-3 \omega^{2}\right]}{(2 j-1)(2 j+3)}\left(-\frac{q^{2} \Delta \alpha}{3 R^{4}}+\frac{q Q}{R^{3}}\right),
\end{aligned}
$$

and

$$
C_{\omega, \omega}^{J, j}(R)=\left\langle J j \omega\left|\hat{\mathbf{I}}^{2} / 2 \mu R^{2}\right| J j \omega\right\rangle=\frac{J(J+1)-2 \omega^{2}+j(j+1)}{2 \mu R^{2}},
$$




$$
\begin{aligned}
C_{\omega, \omega+1}^{J, j} & =\left\langle J j \omega\left|\hat{\mathbf{I}}^{2} / 2 \mu R^{2}\right| J j \omega+1\right\rangle \\
& =\frac{\sqrt{J(J+1)-\omega(\omega+1)} \sqrt{j(j+1)-\omega(\omega+1)}}{\mu R^{2}} .
\end{aligned}
$$

Note that Eqs. (4) represent AC potentials in first order of the PR basis. If necessary, the AC potentials can be corrected by making use of the general "weak field" approximations ${ }^{8-11}$ which use the ratio of the anisotropic part of the interaction to the rotational constant of the molecule as an expansion parameter. In particular, the second-order correction $\Delta V_{\omega}^{j}(R)$ to the PR potentials in Eq. (4) reads

$$
\Delta V_{\omega}^{j}(R)=a_{2}(j, \omega) \frac{1}{B}\left[\frac{3}{2}\left(-\frac{q^{2} \Delta \alpha}{3 R^{4}}+\frac{q Q}{R^{3}}\right)\right]^{2},
$$

where the coefficients $a_{2}(j, \omega)$, as given by Eq. (A4) in Ref. 8 , are

$$
\begin{aligned}
a_{2}(j, \omega)= & -\frac{\left[(j+1)^{2}-\omega^{2}\right]\left[(j+2)^{2}-\omega^{2}\right]}{2(2 j+1)(2 j+3)^{3}(2 j+5)} \\
& +\frac{\left[(j-1)^{2}-\omega^{2}\right]\left(j^{2}-\omega^{2}\right)}{2(2 j-3)(2 j-1)^{3}(2 j+1)} .
\end{aligned}
$$

An interesting feature of the AC potentials is the interplay between the attractive $R^{-4}$ interaction and the attractive/ repulsive $R^{-3}$ interaction. The switching between these two types of interaction occurs at a certain interaction energy. From dimensional considerations we define the characteristic temperature of the low-temperature range as

$$
T_{\mathrm{LT}}=Q^{4} / q^{2} \alpha^{3} k_{\mathrm{B}} .
$$

The temperature $T_{\mathrm{LT}}$ marks the switching of the interaction for partial capture channels $j, \omega$ that affect the partial rate coefficients $k_{j, \omega}$; because of the compensation effects between different $k_{j, \omega}$, the switching in the total rate coefficient $k_{j}$ can occur at substantially lower temperatures. Note also that the two temperatures $T_{\text {rot }}$ and $T_{\mathrm{LT}}$ are not related to each other since they include different parameters (parameters of interaction in $T_{\mathrm{LT}}$ and the molecular constant in $T_{\text {rot }}$ ).

It is generally expected that for not too low collision energies, when the de Broglie wavelength of relative motion $\chi(E)=\hbar / \sqrt{2 \mu E}$ is substantially smaller than the Langevin capture radius $R_{L}(E)=\left(q^{2} \alpha / 2 E\right)^{1 / 4}$, the capture cross section can be calculated in the quasiclassical version of the $\mathrm{AC}$ approximation. The latter, i.e., the $\mathrm{ACCl}$ approximation, describes the relative motion of partners classically and neglects the Coriolis interaction between channels differing in $\omega$. The validity of this approximation was verified earlier by the comparison with classical trajectory calculations in the upper-temperature region of the LT range ${ }^{12}$ where the conservation of the intrinsic angular momentum is still a good approximation. With decreasing collision energies, the $\mathrm{ACCl}$ method is expected to perform well until $\chi(E)$ becomes comparable to $R_{L}(E)$. The relation $\chi(E) \sim R_{L}(E)$ determines the energy where quantum effects become apparent in the capture, and it defines characteristic temperature $T_{\mathrm{VLT}}$ (VLT for very low temperature),

$$
T_{\mathrm{VLT}}=\frac{\hbar^{4}}{k_{\mathrm{B}} \mu^{2} q^{2} \alpha} .
$$

We then conclude that in the temperature range $T_{\mathrm{VLT}}$ $\ll T \ll T_{\text {rot }}$ the capture rate coefficient is adequately reproduced by the ACCl approximation (Sec. III), while in the range $T \ll T_{\mathrm{VLT}}$ the capture rate coefficient is close to its zero-temperature limit (Sec. IV). In the intermediate range, centered at $T=T_{\mathrm{VLT}}$, the capture rate coefficient should be calculated through the solution of the scattering equations (Sec. V).

We consider one more aspect of the statistical description of complex formation at very low temperatures. The current statistical theory of chemical reactions, which operates with the notion of capture probabilities after Bethe, ${ }^{13}$ uses an averaging over the collision energy. ${ }^{14}$ Namely, the cross section is averaged over an energy spread $\delta E$ around the collision energy $E ; \delta E$ is assumed to be small enough compared to the collision energy itself, but big enough to accommodate for many levels of the complex (mean spacing $\Delta \bar{E})$. Within this procedure, the individual Breit-Wigner resonances are regarded as $\delta$ functions centered at resonance energies, and individual widths are replaced by mean widths $\bar{\Gamma}$. For thermal processes one can roughly identify $\delta E$ with $k_{\mathrm{B}} T$, and $\Delta \bar{E}$ with the inverse density of states at the collision threshold $1 / \rho_{c}$. The necessary condition for an applicability of standard type statistical theory, $\delta E \gg \Delta \bar{E}$, can be written as $\rho_{c} k_{\mathrm{B}} T \gg 1$. Clearly this condition is not fulfilled for low temperatures, and the question arises whether the appearance of quantum effects in complex formation (to be discussed in Secs. III-V) is compatible with the restrictive condition $\rho_{c} k_{\mathrm{B}} T \gg 1$. Consider, for instance, the complex $\mathrm{H}_{3}^{+}$, which is assumed to be an intermediate species in the ortho-para conversion of $\mathrm{H}_{2}$ in collisions with protons. The densities of resonance states of $\mathrm{H}_{3}^{+}$have been estimated in Refs. 15 and 16 , and calculated quite accurately for $J=0$ in Ref. 17. According to the latter reference, there are 20 states between the threshold of dissociation of $\mathrm{H}_{3}^{+}$into $\mathrm{H}_{2}(j=0)+\mathrm{H}^{+}$and into $\mathrm{H}_{2}(j=1)+\mathrm{H}^{+}$, with a mean spacing $\Delta \bar{E}_{J=0}$ of about $10 \mathrm{~cm}^{-1}$ between the levels of the same total nuclear spin. Taking as a rough estimate for a rotating complex $\Delta \bar{E}_{J} \approx \Delta \bar{E}_{J=0} /(2 J+1)$, we write the condition $\rho_{c} k_{\mathrm{B}} T \gg 1$ as $k_{\mathrm{B}} T(2 J+1) / \Delta \bar{E}_{J=0} \gg 1$. The quantum effects in the capture rate coefficient show up at about $T=T_{\mathrm{VLT}}$ when the maximal value of $J$ is several units and $T$ is about $T_{\mathrm{VLT}}\left(\mathrm{H}_{2}+\mathrm{H}^{+}\right) \approx 0.04 \mathrm{~K}$. Putting these values into the ratio $k_{\mathrm{B}} T(2 J+1) / \Delta \bar{E}_{J=0}$, we find that the condition $\rho_{c} k_{\mathrm{B}} T \gg 1$ is not fulfilled, implying that the statistical theory in its current version cannot be used for the description of quantum effects in the capture of $\mathrm{H}_{2}$ by $\mathrm{H}^{+}$. One, therefore, should resort either to a more detailed statistical treatment that considers individual Breit-Wigner resonances, or to quantum dynamical calculations such as done in the context of electron-molecule collisions. ${ }^{18}$ This situation changes, however, when the number of atoms in the complex increases. As a qualitative guide, we estimate $\rho_{c}$ within a simple oscillator model as $\rho_{c} \approx(D / \hbar \bar{\omega})^{s}(s !)^{-1}(\hbar \bar{\omega})^{-1}$, where $\bar{\omega}$ is the mean harmonic frequency of the oscillators, $D$ is the 


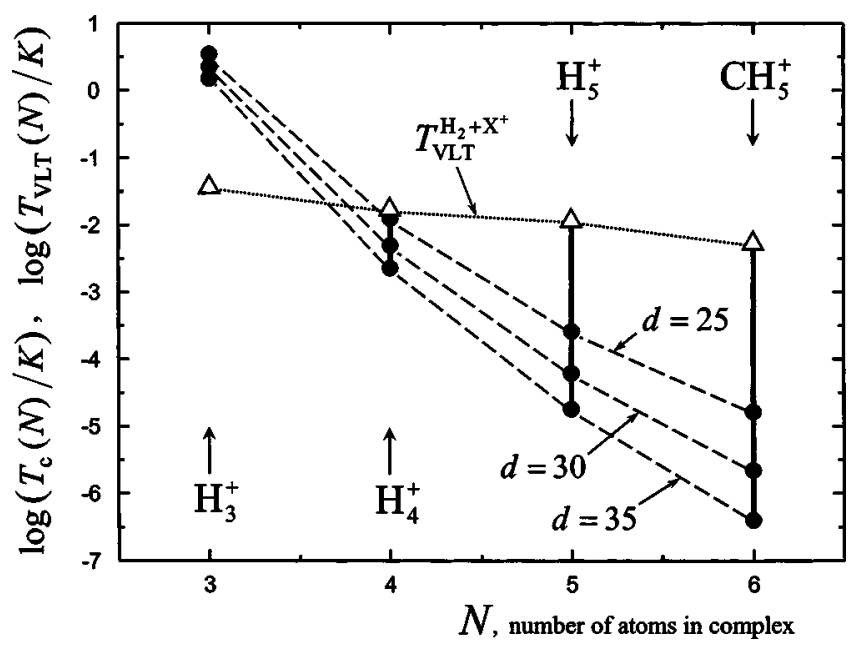

FIG. 1. Characteristic temperature of the level density $T_{c}$ (full circles) and VLT temperature $T_{\mathrm{VLT}}$ (open triangles) vs the number of atoms $N$ in hydrogen-containing complexes, $N=3$ (for $\mathrm{H}_{3}^{+}$), N=4 (for $\mathrm{H}_{4}^{+}$), N=5 (for $\mathrm{H}_{5}^{+}$), and $\mathrm{N}=6$ (for $\mathrm{CH}_{5}^{+}$). The dashed lines connect the points for guiding the eye. The full vertical lines mark the temperature range, where quantum effects of capture are adequately described by conventional statistical theory at different values of the parameter $d=D / \hbar \bar{\omega}$.

dissociation energy of the complex into the reagents, and $s$ $=3 N-7$ with $N$ being the number of the atoms in a complex. ${ }^{19}$ Figure 1 shows the dependence of the mean energy spacing in the complex in temperature units, characteristic temperature of the level density $T_{c}=\Delta \bar{E} / k_{\mathrm{B}}=1 / \rho_{c} k_{\mathrm{B}}$ versus the number of atoms $N$ for $\bar{\omega}=1000 \mathrm{~cm}^{-1}$ and different values of $d=D / \hbar \bar{\omega}$. Values of $N=3,4,5$, and 6 correspond to collisions of $\mathrm{H}_{2}$ with ions $X^{+}=\mathrm{H}^{+}, \mathrm{H}_{2}^{+}, \mathrm{H}_{3}^{+}$, and $\mathrm{CH}_{3}^{+}$, respectively. Note that this estimate yields the correct order of magnitude for $\left.T_{c}(N)\right|_{N=3}=T_{c}\left(\mathrm{H}_{3}^{+}\right)$if one takes the known dissociation energy of $\mathrm{H}_{3}^{+}$about $40000 \mathrm{~cm}^{-1}$. Also shown in this figure are the $T_{\mathrm{VLT}}(N)$ temperatures for the capture of $\mathrm{H}_{2}(j=1)$ by different ions $X^{+}$. Quantum effects in the capture can be described by the statistical approach provided that $T_{c}(N)<T<T_{\mathrm{VLT}}(N)$. We see that, for the collision partners $\mathrm{H}_{2}^{+}, \mathrm{H}_{3}^{+}$, and $\mathrm{CH}_{3}^{+}$, the statistical theory can be used in the region of quantum capture for not too low temperatures $\left[T_{c}(N)<T\right]$. We note also that the temperature $T_{c}$ is extremely sensitive to details of the complex ion model, so that the above estimate of $T_{c}$ through $\rho_{c}$ should be accepted with some caveat.

For the reason of reference, in Table I we list the temperatures $T_{\text {rot }}, T_{\mathrm{LT}}, T_{\mathrm{VLT}}$, and other parameters for two representative systems, $\mathrm{H}_{2}+X^{+}$and $\mathrm{N}_{2}+X^{+}$with a positive ion $X^{+}$of mass of $10 \mathrm{amu}$.

\section{ACCI CAPTURE RATE COEFFICIENTS IN THE LT REGION}

For temperatures well above $T_{\mathrm{VLT}}$, the capture rate coefficients $k_{j}$ can be calculated within the ACCl approach yielding $k_{j}^{\mathrm{ACCl}}$. For the state $j=0, k_{j=0}^{\mathrm{ACCl}}$ is just the temperatureindependent Langevin rate constant $k^{L}$ with a presumably small increment due to a second-order charge-quadrupole correction to the zero-order term in Eq. (4). The value of this correction can be inferred from earlier calculations. For instance, for $\mathrm{H}_{2}+\mathrm{Ar}^{+}$collisions [see Ref. 8, Fig. 1] the fractional increment is below $5 \%$ at $T=T_{\text {rot }}$. For heavier diatoms, it might be higher due to the smaller rotational constant of the diatom. Therefore, we write the expression for the capture rate coefficient $k_{j=0}^{\mathrm{ACCl}}$ as the sum of the Langevin rate coefficient $k^{L}=2 \pi \sqrt{q^{2} \alpha / \mu}$ and an increment $\Delta k_{j=0}^{\mathrm{ACCl}}$, i.e.,

$$
k_{j=0}^{\mathrm{ACCl}}=k^{L}+\Delta k_{j=0}^{\mathrm{ACCl}} .
$$

The increment $\Delta k_{j=0}^{\mathrm{ACCl}}$ is due to a second-order attractive correction to the zero-order channel potential. The former is of a shorter range $\left[\propto R^{-6}\right.$, see Eq. (6)] compared to the latter $\left(\propto R^{-4}\right)$, and therefore $\Delta k_{j=0}^{\mathrm{ACCl}}$ is expected to have a positive temperature dependence. The increment $\Delta k_{j=0}^{\mathrm{ACCl}}$ can be calculated by a perturbation approach such as indicated in Ref. 11.

For $j>0$, in view of the higher spacing between rotational energy levels, the second-order corrections will be lower compared to the case $j=0$. Since the result of the second-order correction to the AC potentials decreases with temperature and since it is completely hidden behind the first-order charge-quadrupole interaction, here we completely neglect it. Thus, for $j>0, k_{j}^{\mathrm{ACCl}}$ is a weighted sum of AC partial capture rate constants $k_{j, \omega}^{\mathrm{ACCl}}$, each calculated for an AC potential $V_{\omega}^{j}$ from Eq. (4):

$$
k_{j}^{\mathrm{ACCl}}=\frac{1}{(2 j+1)} \sum_{\omega=-j}^{j} k_{j, \omega}^{\mathrm{ACCl}} .
$$

For collision energies in the LT regime, some partial capture cross sections vanish when the collision energy becomes lower than the height of the potential barrier, $E_{j, \omega}^{*}$ created by the interplay of the attractive and repulsive parts of the potential which are proportional to $R^{-4}$ and $R^{-3}$, respectively, of the potential. The maximal potential barrier $E_{j, \max }^{*}$ for a given $j$ corresponds either to the channel $\omega=0$ (even case, $q Q>0$ ) or to the channels $\omega= \pm j$ (odd case, $q Q<0$ ). For instance, for $j=1$, the barrier heights in the first approximation are

TABLE I. Characteristic parameters for systems considered in this paper (for a positive ion $X^{+}$of mass $10 \mathrm{amu}$ ): interaction parameters $\alpha$ and $Q$, characteristic temperatures $T_{\text {rot }}, T_{\mathrm{LT}}$, and $T_{\mathrm{VLT}}$, and dimensionless parameters $\varepsilon_{0}$ and $\beta$.

\begin{tabular}{lccccccc}
\hline \hline System & $\alpha$ (a.u.) & $Q($ a.u. $)$ & $T_{\text {rot }}(\mathrm{K})$ & $T_{\mathrm{LT}}(\mathrm{K})$ & $T_{\mathrm{VLT}}(\mathrm{K})$ & $\varepsilon_{0}=T_{\mathrm{LT}} / T_{\text {rot }}$ & $\beta=\mu Q^{2} / \alpha \hbar^{2}$ \\
\hline $\mathrm{H}_{2}+X^{+}$ & 5.44 & 0.474 & 85.3 & 98.8 & $6.20 \times 10^{-3}$ & 1.16 & 126.2 \\
$\mathrm{~N}_{2}+X^{+}$ & 11.7 & -1.09 & 2.88 & 278 & $1.49 \times 10^{-4}$ & 96.5 & 1368 \\
\hline \hline
\end{tabular}


TABLE II. Barrier heights $E_{j=1, \max }^{*}$ (in kelvin) and positions (in atomic units) for initially repulsive AC states for collisions of $\mathrm{H}_{2}(j=1)+$ positive ion $\left(V_{\omega=0}^{j=1} \mathrm{AC}\right.$ potential) and $\mathrm{N}_{2}(j=1)$ + positive ion $\left(V_{\omega=1}^{j=1}\right.$ AC potential) in successive "weak field" approximations. The first entry in each column corresponds to full anisotropic interaction (charge quadrupole + charge- induced dipole), the second entry to charge-quadrupole interaction only.

\begin{tabular}{|c|c|c|c|c|c|c|c|c|}
\hline \multirow{3}{*}{$\begin{array}{l}\text { System } \\
\text { First order }\end{array}$} & \multicolumn{4}{|c|}{$\mathrm{H}_{2}(j=1)+X^{+}, q Q>0$} & \multicolumn{4}{|c|}{$\mathrm{N}_{2}(j=1)+X^{+}, q Q<0$} \\
\hline & \multicolumn{2}{|c|}{$\begin{array}{c}\text { Barrier height } \\
E_{j=1, \max }^{*}(\mathrm{~K})\end{array}$} & \multicolumn{2}{|c|}{$\begin{array}{c}\text { Barrier } \\
\text { position (a.u.) }\end{array}$} & \multicolumn{2}{|c|}{$\begin{array}{c}\text { Barrier height } \\
E_{j=1, \max }^{*}(\mathrm{~K})\end{array}$} & \multicolumn{2}{|c|}{$\begin{array}{c}\text { Barrier } \\
\text { position (a.u.) }\end{array}$} \\
\hline & 1.59 & 2.14 & 21.10 & 19.15 & 0.444 & 0.376 & 33.8 & 35.8 \\
\hline Second order & 1.55 & 2.06 & 21.40 & 19.5 & 0.286 & 0.259 & 41.6 & 42.6 \\
\hline Third order & 1.55 & 2.06 & 21.40 & 19.5 & 0.280 & 0.255 & 42.1 & 43.0 \\
\hline
\end{tabular}

$$
E_{j=1, \max }^{*}=\frac{1}{10}\left(\frac{3}{5}\right)^{3} \frac{1}{q^{2}} \frac{(s Q)^{4}}{(\alpha+4 s \Delta \alpha / 15)^{3}}
$$

where $s=[1+3 \operatorname{sign}(q Q)] / 4$, i.e., $s=1$ for even case and $s=-1 / 2$ for the odd case. The values of the barrier heights for collisions of $\mathrm{H}_{2}+$ positive ion (even case) and $\mathrm{N}_{2}+$ positive ion (odd case) calculated in successive orders are presented in Table II, in which the third order virtually coincides with the accurate AC potentials such as presented in Fig. 1 of Ref. 11. The much lower value of the barrier height for the latter case is related to the small factor $s^{4}=1 / 16$ that appears in Eq. (12) for the odd case. We see that the second-order correction noticeably changes the barrier height for $\mathrm{N}_{2}$-ion collisions. This table also illustrates the convergence of the barrier heights and the barrier positions within the perturbation approach.

One expects that for $T \gg T_{\mathrm{LT}}$, the rate constant $k_{j}^{\mathrm{ACCl}}$ is dominated by the temperature-independent Langevin limit $k^{L}$, while for $T \ll T_{\mathrm{LT}}$, the rate constant $k_{j}^{\mathrm{ACCl}}$ is determined by the attractive charge-quadrupole interaction so that $k_{j}^{\mathrm{ACCl}} \propto T^{-1 / 6}$. For instance, for $j=1$ and the even case, the explicit form of the latter relation reads [Ref. 8, Eq. (22)]

$$
\begin{aligned}
k_{j=1}^{\mathrm{ACCl}} & =\frac{2}{3} k_{j=1, \omega=1}^{\mathrm{ACC}} \\
& \left.\rightarrow \frac{2}{3} \Gamma(1 / 3) \sqrt{\frac{\pi}{\mu}}\left(\frac{k_{\mathrm{B}} T}{2}\right)^{-1 / 6}\left|\frac{2}{5} q Q\right|^{2 / 3}\right|_{T \ll T_{\mathrm{LT}}} .
\end{aligned}
$$

Since all $\mathrm{ACCl}$ rate coefficients display the same dependence on the reduced mass (inversely proportional to $\sqrt{\mu}$ ), it is expedient to introduce the mass-independent reduced rate coefficient $\chi_{j}^{\mathrm{ACCl}}(T)=k_{j}^{\mathrm{ACCl}}(T) / k^{L}$ which applies to all the collisions studied in this work. Figure 2 shows the first-order reduced rate coefficients $\chi_{j=0}^{\mathrm{ACCl}}$ and $\chi_{j=1}^{\mathrm{ACCl}}$ (with the partial contributions $\chi_{j=1, \omega}^{\mathrm{ACCl}}$ ) across the LT range as a function of the reduced temperature $T / T^{*}, T^{*}=E_{j, \max }^{*} / k_{\mathrm{B}}$ for $\mathrm{H}_{2}+$ ion capture. This figure is similar to Fig. 1 from Ref. 8, save for a different scaling of the temperature that emphasizes the opening of the channel with a barrier and a logarithmic abscissa chosen to better reproduce the extreme left of the LT range. A rather unexpected feature in this figure is the quite small deviation of $\chi_{j=1}^{\mathrm{ACCl}}$ from unity for temperatures close to and above $T^{*}$ although partial coefficients $\chi_{j=1, \omega}^{\mathrm{ACCl}}$ are quite far from their asymptotic values. A shallow minimum of $\chi_{j=1}^{\mathrm{ACCl}}$ originates from the interplay of capture in two adiabatic channels. The two characteristic temperatures $T_{\mathrm{rot}}$ and $T_{\mathrm{VLT}}$ are marked in Fig. 2; the latter depends on the collision partner which here corresponds to $\mathrm{H}_{2}+\mathrm{H}_{3}^{+}$collisions.

In the attempt to provide more general results for iondiatom capture rate coefficients in terms of a small number of parameters and for arbitrary signs of the product $q Q$, we neglect the anisotropy of polarizability of the diatom $\Delta \alpha$ and introduce a scaling of the energy and the distance,

$$
E=E_{0} \varepsilon \quad \text { with } E_{0}=Q^{4} / q^{2} \alpha^{3}
$$

and

$$
R=R_{0} \rho \quad \text { with } R_{0}=\alpha|q / Q| .
$$

The scaled AC potentials in first order contain no parameters and can be written as

$$
v_{0}^{0}(\rho)=V_{0}^{0}(R) / E_{0}=-\frac{1}{2 \rho^{4}}
$$

and

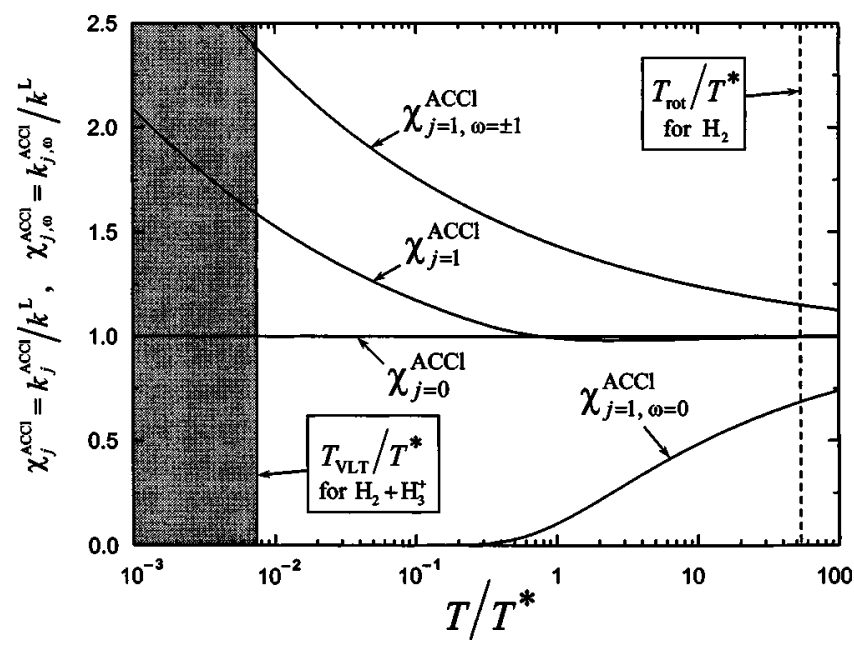

FIG. 2. Partner-independent total and partial scaled ACCl rate coefficients for $\mathrm{H}_{2}+$ ion capture, $\chi_{j=1}^{\mathrm{ACCl}}=k_{j=1}^{\mathrm{ACCl}} / k^{L}$ and $\chi_{j=1, \omega}^{\mathrm{ACCl}}=k_{j=1, \omega}^{\mathrm{ACCl}} / k^{L}$, respectively, vs reduced temperature $T / T^{*}$ across the LT range ( $T^{*}$ from Sec. III). The dashed vertical line at the right-hand side of the figure corresponds to $T$ $=T_{\text {rot }}$ and the full vertical line at the left-hand side corresponds to $T=T_{\mathrm{VLT}}$ for $\mathrm{H}_{2}+\mathrm{H}_{3}^{+}$collisions. For $T>T_{\text {rot }}$, the perturbed-rotor approximation may not be valid and for $T<T_{\mathrm{VLT}}$ (the shaded area) the $\mathrm{ACCl}$ approximation is not expected to be adequate. Note that the scaling factor for the temperature $1 / T^{*}$, is the same for different collision partners. 


$$
v_{\omega}^{j, \pm}(\rho)=V_{\omega}^{j, \pm}(R) / E_{0}=-\frac{1}{2 \rho^{4}} \pm \frac{\left[j(j+1)-3 \omega^{2}\right]}{(2 j-1)(2 j+3)} \frac{1}{\rho^{3}},
$$

where the plus and minus signs correspond to the even and odd cases, respectively. The second-order correction depends only on one dimensionless parameter $\varepsilon_{0}=E_{0} / B=T_{\mathrm{LT}} / T_{\text {rot }}$ and reads

$$
\Delta v_{\omega}^{j}\left(\rho, \varepsilon_{0}\right)=\frac{9}{4} a_{2}(j, \omega) \frac{\varepsilon_{0}}{\rho^{6}},
$$

where $a_{2}(j, \omega)$ are given by Eq. (7). For instance, the explicit expression for $j=0$ and $\omega=0$ reads $\Delta v_{0}^{0}\left(\rho, \varepsilon_{0}\right)=-\varepsilon_{0} / 30 \rho^{6}$.

For the potentials of Eq. (15), the reduced rate coefficients can be represented as the integral (see the Appendix)

$$
\begin{aligned}
\chi_{j, \omega}^{\mathrm{ACCl}, \pm}(\theta)= & \frac{\theta^{-3 / 2}}{2 \sqrt{2 \pi}} \int_{0}^{\infty} \rho^{3}\left(v_{\omega}^{j, \pm}\right)^{\prime} \\
& \times\left[-\rho\left(v_{\omega}^{j, \pm}\right)^{\prime \prime}-3\left(v_{\omega}^{j, \pm}\right)^{\prime}\right] \Theta\left[\left(v_{\omega}^{j, \pm}\right)^{\prime}\right] \\
& \times \exp \left\{-\left[\rho\left(v_{\omega}^{j, \pm}\right)^{\prime}+2 v_{\omega}^{j, \pm}\right] / 2 \theta\right\} d \rho
\end{aligned}
$$

and

$$
\chi_{j}^{\mathrm{ACCl}, \pm}(\theta)=\frac{1}{(2 j+1)} \sum_{\omega=-j}^{\omega=j} \chi_{j, \omega}^{\mathrm{ACCl}, \pm},
$$

where the integrand functions are: $v_{\omega}^{j, \pm} \equiv v_{\omega}^{j, \pm}(\rho), \quad\left(v_{\omega}^{j, \pm}\right)^{\prime}$ $\equiv d v_{\omega}^{j, \pm}(\rho) / d \rho, \quad\left(v_{\omega}^{j, \pm}\right)^{\prime \prime} \equiv d^{2} v_{\omega}^{j, \pm}(\rho) / d \rho^{2}, \Theta$ is the Heaviside step function, and $\theta$ is the reduced temperature $\theta=T / T_{L T}$.

For large values of $j$, Eq. (15) can be rewritten as

$$
\left.v_{\omega}^{j, \pm}(\rho)\right|_{j \gg 1}=v^{ \pm}(\rho, x)=-\frac{1}{2 \rho^{4}} \pm \frac{\left(1-3 x^{2}\right)}{4 \rho^{3}},
$$

with $x=\omega / j$. Then the total rate coefficient is expressed as

$$
\begin{aligned}
\chi_{j \gg 1}^{\mathrm{ACCl} 1 \pm}(\theta)= & \frac{\theta^{-3 / 2}}{\sqrt{2 \pi}} \int_{0}^{1} d x \int_{0}^{\infty} \rho^{3}\left(v^{ \pm}\right)^{\prime}\left[-\rho\left(v^{ \pm}\right)^{\prime \prime}\right. \\
& \left.-3\left(v^{ \pm}\right)^{\prime}\right] \Theta\left[\left(v^{ \pm}\right)^{\prime}\right] \\
& \times \exp \left\{-\left[\rho\left(v^{ \pm}\right)^{\prime}+2 v^{ \pm}\right] / 2 \theta\right\} d \rho,
\end{aligned}
$$

with the functions of the integrand $v^{ \pm} \equiv v^{ \pm}(\rho, x), \quad\left(v^{ \pm}\right)^{\prime}$ $\equiv d v^{ \pm}(\rho, x) / d \rho$, and $\left(v^{ \pm}\right)^{\prime \prime} \equiv d^{2} v^{ \pm}(\rho, x) / d \rho^{2}$.

Figure 3 compares first-order $\mathrm{ACCl}$ capture rates for different rotational quantum numbers in the even case $q Q>0$ (superscript "+"). It is seen that, with increase in $j$, the $\chi_{j}^{\mathrm{ACCl},+}$ converge to a limiting curve which can be considered as the fully classical $\mathrm{AC}$ limit $(j \gg 1)$ of the state-specific (fixed $j$ ) low-temperature $\left(T \ll T_{\text {rot }}\right)$ capture rate constant, such as obtained when both the relative motion and molecular rotation are treated classically. Similar results for odd case $q Q<0$ (superscript "-") are shown in Fig. 4.

The given results can also be used for the illustration on the capture rates of the effects of the polarization anisotropy and higher-order charge-quadrupole interactions. As an example we take collisions of $\mathrm{N}_{2}(j=0,1)$ with positive (the odd case) and negative (even case) ions, which is a quite unfavorable case for the application of the weak field approximation because of the rather small value of $B$. At the beginning we note that, although the height of the potential

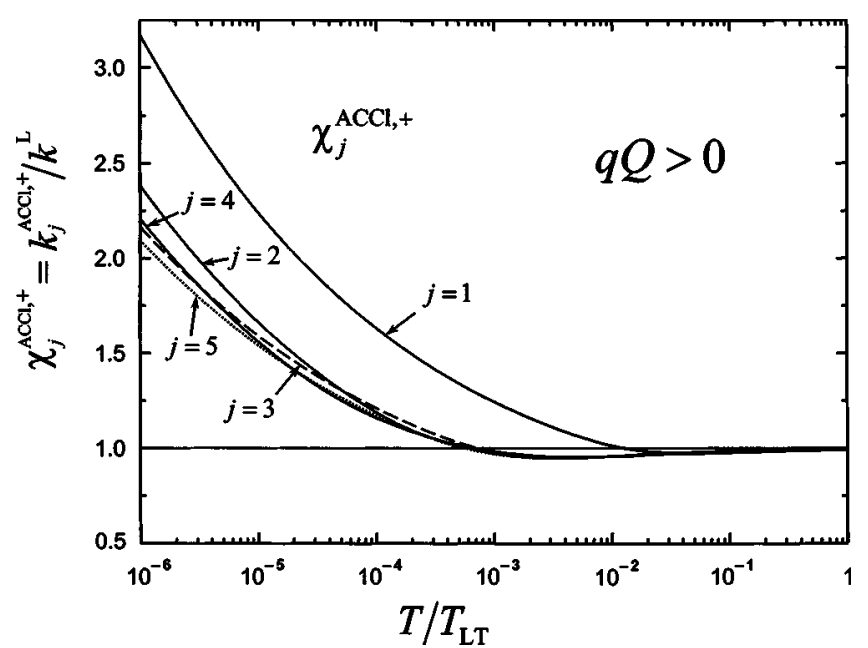

FIG. 3. Universal scaled first-order $\mathrm{ACCl}$ capture rate coefficients $\chi_{j}^{\mathrm{ACCl},+}$ for homonuclear diatomics in the even case $(q Q>0)$ vs reduced temperature $T / T_{\mathrm{LT}}$ across the LT range in first-order PR approximation (with polarizability anisotropy neglected). Note that the scaling factor for the temperature $1 / T_{\mathrm{LT}}$ is the same for different collision partners.

barrier $E_{j, \omega}^{*}$ for $j=1$ is a sensitive function of the polarization anisotropy (see Table II), the latter only insignificantly affects the total rate coefficients. This is related to the fact that inclusion of the polarization anisotropy affects the partial rate coefficients in opposite directions. Therefore, we will disregard it. However, the situation is different for the second-order correction to the interaction potential, which affects all partial rates of $j=1$ in the same direction [see Eqs. (4) and (7) for $a_{2}(j, \omega)$.

The plots of the capture rate coefficients for $j=1$, for both even and odd cases are calculated with the first-order PR potentials for $\mathrm{N}_{2}-q^{+}$and $\mathrm{N}_{2}-q^{-}$systems without polarization anisotropy and shown in Fig. 5. Also given are the capture rate coefficients for $j=0$ in second order and the points for the same systems from Table III of Ref. 11 such as calculated with accurate AC potentials for $T=0.5,1,2,4 \mathrm{~K}$. We see that for, $j=0$, the second-order capture rate coefficient $\chi_{j=0}^{\mathrm{ACCl}, \pm}$ smoothly passes through the data points from Ref. 11, averaged between even and odd cases in order to

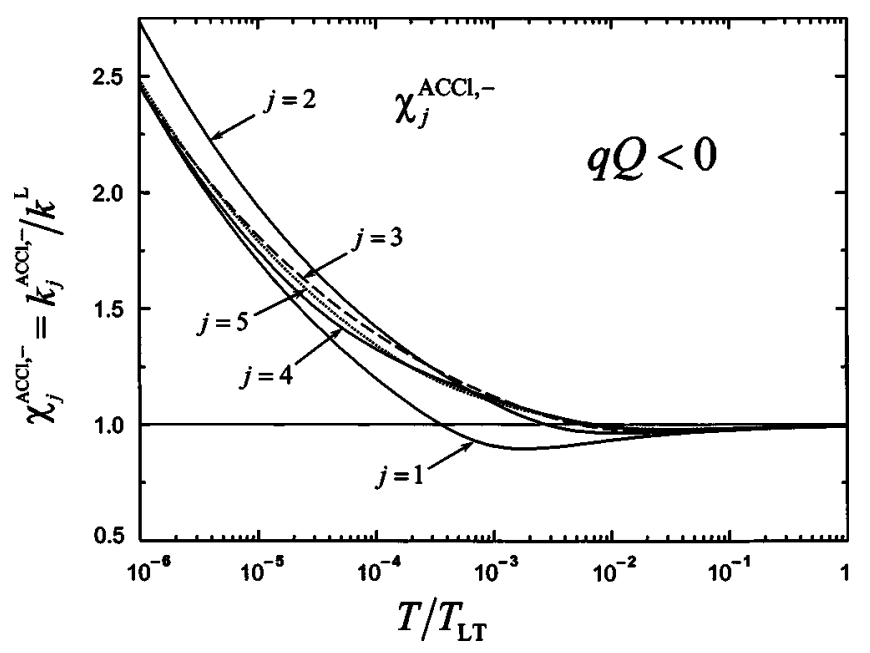

FIG. 4. The same as in Fig. 3 but in the odd case $(q Q<0)$. 


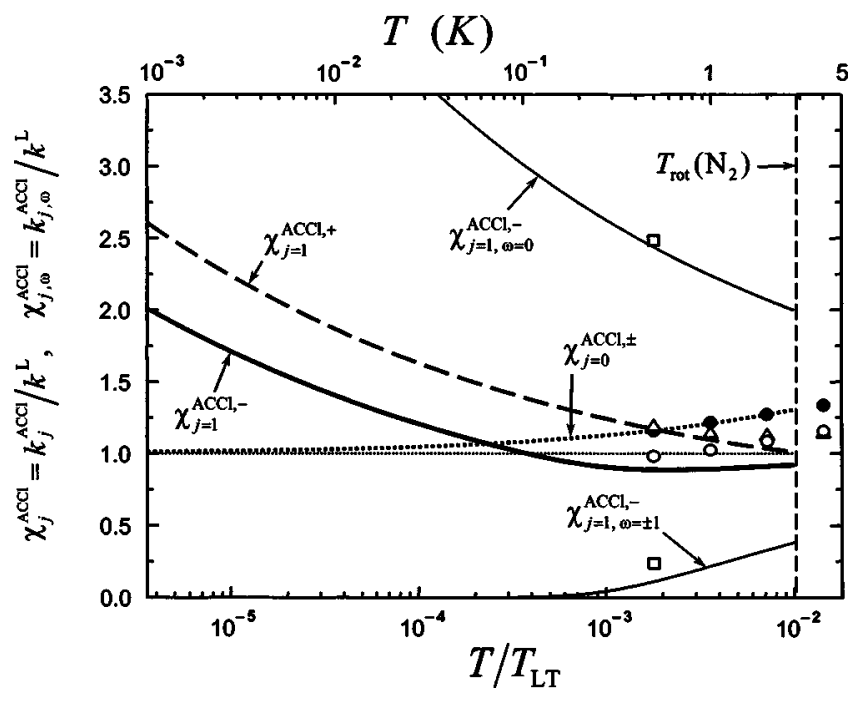

FIG. 5. Comparison of ACCl capture rate coefficients for $\mathrm{N}_{2}(j=0,1)+X^{\overline{ }}$ collisions (the even case corresponds to a negative ion and the odd case to a positive one) with accurate $\mathrm{ACCl}$ results from Ref. 11. The dashed vertical line corresponds to $T=T_{\text {rot }}\left(\mathrm{N}_{2}\right)=2.88 \mathrm{~K}$. The heavy solid line corresponds to first-order $\chi_{j=1}^{\mathrm{ACCl},-}$, the light solid lines to its partial components $\chi_{j=1, \omega=0}^{\mathrm{ACCl},-}$ and $\chi_{j=1, \omega= \pm 1}^{\mathrm{ACCl},-}$ in first order, the heavy dashed linecorresponds to first-order $\chi_{j=1}^{\mathrm{ACCl},+}$, and the heavy dots to $\chi_{j=0}^{\mathrm{ACCl}}$ in second order. The open triangles and circles give the accurate results in the even and odd cases for $j=1$ and the filled circles represent the averaged odd-even case for $j=0$ from Ref. 11 for four temperatures, $T=0.5,1,2,4 \mathrm{~K}$, respectively. Also shown are accurate partial rate coefficients for the odd $j=1$ case at $T=0.5 \mathrm{~K}$ (open squares).

eliminate third-order effects. For $j=1$, the first-order rate coefficient $\chi_{j=1}^{\mathrm{ACCl},+}$ nicely hits the first point from Ref. 11 (triangle), implying that at this point and to the left of it the first-order PR approximation performs well. To the right of this point, the first-order approximation performs progressively worse, such as follows from the comparison of our graph the data points from Ref. 11. This is expected on the basis of a comparison of the points at $T=1,2,4 \mathrm{~K}$ while the rotational temperature $T_{\text {rot }}\left(\mathrm{N}_{2}\right)$ equals $2.88 \mathrm{~K}$. The odd case, for $j=1$, is different such as noticed by the shift of the firstorder plot of $\chi_{j=1}^{\mathrm{ACCl},-}$ relative to the first accurate point at $T$ $=0.5 \mathrm{~K}$. In order to elucidate the origin of this discrepancy, we also present the plots of the partial rate coefficients $\chi_{j=1, \omega}^{\mathrm{ACCl},-}$. We see that the discrepancy is due largely to the underestimation of the rate coefficient for the capture channel with the activation barrier. As follows from Table II, the height of the activation barrier for the odd case diminishes quite noticeably when one goes to higher-order perturbation terms. Actually, if one calculates the partial rate coefficient $\chi_{j=1, \omega= \pm 1}^{\mathrm{ACCl},-}$ in third order, the respective plot passes through the first point. The same correction only slightly modifies the plot of $\chi_{j=1, \omega=0}^{\mathrm{ACCl},}$. This shows that at $T=0.5 \mathrm{~K}$, the high-order corrections to the AC potentials for $\mathrm{N}_{2}+q$ collision are important; however, it is also clear that with decrease in temperature the first-order PR approximation will become adequate simply because the contribution from the $j=1$, $\omega= \pm 1$ channel disappears, like in the case with $j=1, \omega=0$ channel for the even case at substantially higher temperatures. Because of the higher rotational temperature $\left[T_{\text {rot }}\left(\mathrm{H}_{2}\right)=85.3 \mathrm{~K}\right]$, this kind of discrepancy will be much smaller for the capture of $\mathrm{H}_{2}$ which is characterized by a better applicability of the first-order charge-dipole approximation.

\section{ZERO-TEMPERATURE LIMIT OF THE CAPTURE RATE COEFFICIENTS}

For temperatures well below $T_{\mathrm{VLT}}$, the capture rate coefficients at $T \rightarrow 0$ approaches, according to the BetheWigner law, ${ }^{20}$ constant values, denoted by $\lim _{T \rightarrow 0} k_{j}(T)=k_{j}^{\mathrm{B}}$ (superscript "B" stands for Bethe). Any difference between $\mathrm{ACCl}$ and accurate quantum rates, which arises in this temperature range, is due to the following simplifications inherent in the ACCl treatment: the neglect of quantization of the relative and total angular momenta, the disregard of overbarrier reflection and underbarrier transmission, and the omission of the Coriolis coupling between AC states for $j>0$.

For $j=0$, the value of $k_{j=0}^{\mathrm{B}}$ can be derived from the solution of the $s$-wave zero-energy Schrödinger equation for capture with a central potential $V_{\omega=0}^{j=0}$. To the second order in the PR basis, which includes the lowest order of the chargequadrupole interaction, the potential reads

$$
V_{\omega=0}^{j=0}(R)=-\frac{\alpha q^{2}}{2 R^{4}}-\frac{1}{30} \frac{q^{2} Q^{2}}{B R^{6}} .
$$

The zero-energy capture solution for this potential is known, ${ }^{21}$ leading to the following expression for the Bethe rate coefficient:

$$
k_{j=0}^{\mathrm{B}}=2 k^{\mathrm{L}} F\left(\sqrt{\varepsilon_{0} / 5 \beta}\right) .
$$

Here $\varepsilon_{0}=E_{0} / B=Q^{4} / q^{2} \alpha^{3} B$ is the dimensionless parameter already introduced earlier, $\beta=Q^{2} \mu / \alpha \hbar^{2}$ is yet another dimensionless parameter and the function $F(x)$ is given by ${ }^{21}$

$$
\begin{aligned}
F(x)= & \left(\frac{x}{\sqrt{3}}\right)^{1 / 2} \frac{\Gamma(1 / 2)}{\Gamma(3 / 2)}\left|\frac{\Gamma(3 / 4-i \sqrt{3} / 4 x)}{\Gamma(1 / 4-i \sqrt{3} / 4 x)}\right| \\
& \times \sin \left[\frac{\pi}{4}-\arg \left(\frac{\Gamma(3 / 4-i \sqrt{3} / 4 x)}{\Gamma(1 / 4-i \sqrt{3} / 4 x)}\right)\right] .
\end{aligned}
$$

In the limit of vanishing charge-quadrupole interaction, $\varepsilon_{0} / \beta \rightarrow 0, F$ tends to unity, and Eq. (21) reduces to the VogtWannier result ${ }^{22} k_{j=0}^{\mathrm{B}}=2 k^{\mathrm{L}}$ discussed also in Refs. 18 and 23. For instance, for capture of $\mathrm{N}_{2}$ by an ion of mass $10 \mathrm{amu}$, we have $\varepsilon_{0}=96.5, \beta=1368$, i.e., $\sqrt{\varepsilon_{0}} / 5 \beta=0.12$, such that the correction to the Vogt-Wannier result is quite small (amounting to less then $1 \%$ ). For the capture of $\mathrm{H}_{2}$, it is even smaller.

For $j>0$, the value of $k_{j}^{\mathrm{B}}$ should be found from the solution of the coupled zero-energy Schrödinger equation in the total angular momentum representation for a manifold corresponding to the $|J j \omega\rangle$ states with $J=j$. We have recently shown, for the $j=1$ state that this solution can be well approximated by the solution of uncoupled equations in the so-called axially nonadiabatic channel basis. ${ }^{4}$ Within this approach, the zero-energy capture is described by a single equation that contains the attractive effective potential $V_{a}^{j}(R)$ which, in turn, is the lowest eigenvalue of the matrix composed of the first-order AC potential and the Coriolis interaction between different $\omega$ states. Now we introduce yet another approximation that allows one to calculate $V_{a}^{j}(R)$ 
analytically in the perturbative approach. The justification for this approximation is the following. Capture in the limit of zero energies is dominated by the channel with orbital quantum number $\ell=0$. Though $\ell$ is not an exact quantum number and has only asymptotic significance, it can be considered as a good quantum number in the limit of zero collision energies since capture occurs at very large distances. One can thus calculate the effective potential in the $s$ channel using the perturbation approach in the basis of the asymptotic functions in the $j \ell J$ representation. The perturbative approximation to $V_{a}^{j}(R)$, i.e., $V_{a \text {,pert }}^{j}(R)$, which is valid up to the second order, reads

$$
\begin{aligned}
V_{a, \text { pert }}^{j}(R)= & \left.\langle\mathrm{Jj} \ell|U(R, \gamma)| J j \ell\rangle\right|_{\substack{J=j=1 \\
\ell=0},} \\
& -\left.\frac{\left\langle J j \ell|U(R, \gamma)| J j \ell^{\prime}\right\rangle^{2}}{\Delta E_{\ell \ell^{\prime}}(R)}\right|_{\substack{J=j=1 \\
\ell=0 \\
\ell^{\prime}=2}},
\end{aligned}
$$

where $\Delta E_{02}(R)$ is the difference between the relative rotational energy for $\ell=0$ and $\ell^{\prime}=2$ orbital states:

$$
\Delta E_{02}(R)=6 \hbar^{2} / 2 \mu R^{2}
$$

Using the standard formula ${ }^{24}$

$$
\left\langle j \ell J\left|P_{2}(\cos \gamma)\right| j \ell^{\prime} J\right\rangle{\substack{\ell=J \\ \ell=0 \\ \ell^{\prime}=2}}_{j=}=\frac{-1}{\sqrt{5}} \sqrt{\frac{j(j+1)}{(2 j-1)(2 j+3)}},
$$

we write

$$
\begin{aligned}
& V_{a, \mathrm{pert}}^{j}(R)=-\frac{q^{2} \alpha^{j, \mathrm{eff}}}{2 R^{4}}, \\
& \alpha^{j, \mathrm{eff}}=\alpha\left(1+\frac{2 \beta}{15} \frac{j(j+1)}{(2 j-1)(2 j+3)}\right) .
\end{aligned}
$$

We thus see that the anisotropic interaction decreasing as $R^{-3}$ is modified into an isotropic interaction decreasing as $R^{-4}$ which results from the gyroscopic nature of the rotational motion. In other words, we can say that the strong Coriolis coupling appearing in the $j \omega J$ representation modifies the first-order charge-quadrupole interaction, making it of a shorter range $\left(\propto 1 / R^{4}\right.$ instead of $\left.\propto 1 / R^{3}\right)$. The net result of this modification is the replacement of $\alpha$ by $\alpha^{j \text {,eff }}$ in the expression for $k^{L}$, see Eq. (10), which results in the following expression for zero-temperature limit:

$$
\begin{aligned}
k_{j}^{\mathrm{B}} & \approx k_{j, \mathrm{pert}}^{\mathrm{B}}=2 k^{\mathrm{L}} \sqrt{\frac{\alpha^{j, \mathrm{eff}}}{\alpha}} \\
& =2 k^{\mathrm{L}} \sqrt{1+\frac{2 \beta}{15} \frac{j(j+1)}{(2 j-1)(2 j+3)}} .
\end{aligned}
$$

We see that the zero-temperature limit of the capture rate coefficient in the perturbative approach $k_{j \text {,pert }}^{\mathrm{B}}$ does not depend on the sign of $q Q$, i.e., for a given $j$, it is the same for even and odd cases.

In the limit of prevailing charge-quadrupole interaction Eq. (27) yields

$$
k_{j, \mathrm{pert}}^{\mathrm{B}}=\frac{4 \pi|q Q|}{\hbar} \sqrt{\frac{2 j(j+1)}{15(2 j-1)(2 j+3)}} .
$$

Though the interaction that leads to Eq. (28) is of Langevintype (proportional to $R^{-4}$ ), the functional form of this expression is the same as expected from an effective isotropic interaction of the form $-\lambda|q Q| / R^{3}$, where $\lambda$ is a certain numerical coefficient. The value of $\lambda$ can be found from the comparison of Eq. (28) with the Bethe expression for the rate coefficient for the potential $-\lambda|q Q| / R^{3}$ from Ref. 23 which yields $\lambda \approx 1 / 10 \pi \approx 0.03$. We see that this value of $\lambda$ is much smaller than the coefficient that corresponds to maximum attraction for $j=1(1 / 5$ for the even case and $2 / 5$ for the odd case) or $j \gg 1$ (1/2 for the even case and $1 / 4$ for the odd case), see Eq. (4). The small value of $\lambda$ reflects the effect of the partial averaging of the charge-quadrupole interaction.

\section{QUALITATIVE BEHAVIOR OF CAPTURE RATE CONSTANTS $k_{j}$ ACROSS THE VLT RANGE AND ACCURATE RESULTS FOR $k_{j=0}$ AND $k_{j=1}$}

Comparing Eqs. (10) and (13) with Eqs. (21) and (27) one sees that, with decrease in the temperature, the accurate rate constant for $j=0$ exceeds its $\mathrm{ACCl}$ counterpart while the opposite is true for $j>0$. This means that, within the VLT range, the accurate capture rates for $j=0$ and $j>0$ are closer to each other than given within the $\mathrm{ACCl}$ approach. The reason for this is the partial averaging of the anisotropic interaction as a result of the Coriolis coupling between $\mathrm{AC}$ potentials, or, in other words, by the rotation of the collision axis. This explains why $k_{j}^{\mathrm{B}}$ increases with $\mu$ : the rotation of the collision axis, which persists even at zero collision energy because of the coupling of the relative and intrinsic angular momenta, is slower for heavier partners; this results in a less efficient averaging of the $1 / R^{3}$ potential and an increase of the rate coefficient. Our analysis shows that, in the VLT regime, one can speak of a quantum amplification of the $\mathrm{ACCl}$ rate coefficient for $j=0$ and a quantum suppression of the $\mathrm{ACCl}$ rate coefficient for $j>0$.

The transition from the Bethe to the $\mathrm{ACCl}$ limits can be accomplished by solving the coupled quantum equations that describe capture. The explicit form of the equations and the boundary conditions, were discussed in Ref. 3. For different homonuclear diatom+ion collisions these equations in the dimensionless form differ, for the first-order PR potentials, only in the values of the parameter $\beta=\mu Q^{2} / \alpha \hbar^{2}$ and in the sign of the product $q Q$.

In what follows, we will consider collisions of $\mathrm{H}_{2}, \mathrm{HD}$, and $\mathrm{D}_{2}$ with hydrogen-containing ions (the even case, $q Q>0$ ) within the first-order PR approximation. The capture rate constants for $j>1$ as functions of a properly scaled temperature, are qualitatively similar to the rate coefficients for $j=1$. Therefore, we consider the cases $j=0$ and $j=1$. In our numerical calculations the range of the collision energies was taken from a region corresponding to a linear dependence of the capture probability on to the wave vector (Bethe limit) and the region where the accurate capture rate coefficients converged to their $\mathrm{ACCl}$ counterparts. Within our error limits, this convergence was found to be satisfactory, before the capture in the $\omega=0 \mathrm{AC}$ channel becomes noticeable. We 
TABLE III. Langevin capture rate coefficients (in $10^{-9} \mathrm{~cm}^{3}$ molecule $\mathrm{e}^{-1} \mathrm{~s}^{-1}$ ) and characteristic parameters entering into the expressions for amplification and suppression factors, Eqs. (31) and (32).

\begin{tabular}{cllcccc}
\hline \hline No. & Collision & $k^{L}$ & $T_{\mathrm{VLT}}\left(10^{-3} \mathrm{~K}\right)$ & $\beta=\mu Q^{2} / \alpha \hbar^{2}$ & $n$ & $k_{j=1}^{\mathrm{B}} / k^{L}\left(k_{j=1, \mathrm{pert}}^{\mathrm{B}} / k^{L}\right)$ \\
\hline 1 & $\mathrm{H}_{2}+\mathrm{H}_{2}^{+}$ & 2.10 & 17.2 & 75.80 & 3.3 & $4.28(4.49)$ \\
2 & $\mathrm{HD}+\mathrm{H}_{2}^{+}$ & 1.91 & 12.0 & 90.95 & 3.2 & $4.60(4.84)$ \\
3 & $\mathrm{D}_{2}+\mathrm{H}_{2}^{+}$ & 1.81 & 9.70 & 101.0 & 3.2 & $4.80(5.06)$ \\
4 & $\mathrm{H}_{2}+\mathrm{HD}^{+}$ & 1.91 & 12.0 & 90.95 & 3.2 & $4.60(4.84)$ \\
5 & $\mathrm{HD}^{+} \mathrm{HD}^{+}$ & 1.71 & 7.65 & 113.6 & 3.1 & $5.06(5.32)$ \\
6 & $\mathrm{D}_{2}+\mathrm{HD}^{+}$ & 1.60 & 5.86 & 129.8 & 3.1 & $5.36(5.63)$ \\
7 & $\mathrm{H}_{2}+\mathrm{D}_{2}^{+}$ & 1.81 & 9.70 & 101.0 & 3.2 & $4.80(5.06)$ \\
8 & $\mathrm{HD}_{2}+\mathrm{D}_{2}^{+}$ & 1.60 & 5.86 & 129.8 & 3.1 & $5.36(5.63)$ \\
9 & $\mathrm{D}_{2}+\mathrm{D}_{2}^{+}$ & 1.48 & 4.30 & 151.5 & 3.1 & $5.74(6.03)$ \\
10 & $\mathrm{H}_{2}+\mathrm{H}_{3}^{+}$ & 1.91 & 12.0 & 90.95 & 3.2 & $4.60(4.84)$ \\
11 & $\mathrm{HD}^{+} \mathrm{H}_{3}^{+}$ & 1.71 & 7.65 & 113.6 & 3.1 & $5.06(5.32)$ \\
12 & $\mathrm{D}_{2}+\mathrm{H}_{3}^{+}$ & 1.60 & 5.86 & 129.8 & 3.1 & $5.36(5.63)$ \\
13 & $\mathrm{H}_{2}+\mathrm{H}_{2} \mathrm{D}^{+}$ & 1.81 & 9.70 & 101.0 & 3.2 & $4.80(5.06)$ \\
14 & $\mathrm{HD}^{+} \mathrm{H}_{2} \mathrm{D}^{+}$ & 1.60 & 5.86 & 129.8 & 3.1 & $5.36(5.63)$ \\
15 & $\mathrm{D}_{2}+\mathrm{H}_{2} \mathrm{D}^{+}$ & 1.48 & 4.30 & 151.5 & 3.1 & $5.74(6.03)$ \\
16 & $\mathrm{H}_{2}+\mathrm{HD}_{2}^{+}$ & 1.75 & 8.43 & 108.2 & 3.1 & $4.96(5.20)$ \\
17 & $\mathrm{HD}^{+} \mathrm{HD}_{2}^{+}$ & 1.53 & 4.90 & 142.0 & 3.1 & $5.56(5.86)$ \\
18 & $\mathrm{D}_{2}+\mathrm{HD}_{2}^{+}$ & 1.40 & 3.48 & 168.3 & 3.0 & $6.02(6.32)$ \\
19 & $\mathrm{H}_{2}+\mathrm{D}_{3}^{+}$ & 1.71 & 7.65 & 113.6 & 3.1 & $5.06(5.32)$ \\
20 & $\mathrm{HD}_{2}+\mathrm{D}_{3}^{+}$ & 1.48 & 4.30 & 151.5 & 3.1 & $5.74(6.03)$ \\
21 & $\mathrm{D}_{2}+\mathrm{D}_{3}^{+}$ & 1.35 & 3.00 & 181.8 & 3.0 & $6.24(6.55)$ \\
22 & $\mathrm{H}_{2}+\mathrm{CH}_{3}^{+}$ & 1.58 & 5.53 & 133.7 & 3.1 & $5.43(5.70)$ \\
23 & $\mathrm{HD}_{2}+\mathrm{CH}_{3}^{+}$ & 1.33 & 2.75 & 189.5 & 3.0 & $6.35(6.67)$ \\
24 & $\mathrm{D}_{2}+\mathrm{CH}_{3}^{+}$ & 1.18 & 1.73 & 239.3 & 3.0 & $7.07(7.42)$ \\
25 & $\mathrm{H}_{2}+\mathrm{C}_{2} \mathrm{H}_{2}^{+}$ & 1.54 & 5.00 & 140.8 & 3.1 & $5.55(5.83)$ \\
26 & $\mathrm{HD}_{2}+\mathrm{C}_{2} \mathrm{H}_{2}^{+}$ & 1.28 & 2.38 & 204.0 & 3.0 & $6.56(6.89)$ \\
27 & $\mathrm{D}_{2}+\mathrm{C}_{2} \mathrm{H}_{2}^{+}$ & 1.12 & 1.43 & 262.8 & 3.0 & $7.38(7.75)$ \\
\hline \hline & & & & & &
\end{tabular}

therefore do not consider quantum effects that arise in the region of classical opening of the AC with $\omega=0$.

The most economical representation of accurate numerical results is given by two functions, the amplification and suppression factors, which are defined as

$$
\begin{aligned}
& A(T)=k_{j=0}(T) / k_{j=0}^{\mathrm{ACCl}}(T), \\
& S(T)=k_{j=1}(T) / k_{j=1}^{\mathrm{ACCl}}(T),
\end{aligned}
$$

respectively and which possess the following asymptotic properties:

$$
\begin{gathered}
A(T)=\left\{\begin{array}{cc}
1, & T \gg T_{\mathrm{VLT}} \\
k_{j=0}^{\mathrm{B}} / k^{L}=2 & T \rightarrow 0,
\end{array}\right. \\
S(T)=\left\{\begin{array}{cc}
1, & T \gg T_{\mathrm{VLT}} \\
k_{j=1}^{\mathrm{B}} / k_{j=1}^{\mathrm{ACCl}}(T), & T \rightarrow 0 .
\end{array}\right.
\end{gathered}
$$

The optimal results for $A(T)$ and $S(T)$ were found to be the following.

Amplification factor. The suggested expression for the amplification factor is

$$
\begin{gathered}
A(T)=\frac{3}{2}-\frac{1}{2} \tanh \left[0.925 \zeta+0.18 \zeta^{2}+0.04 \zeta^{3}\right], \\
\zeta=\log _{10}\left(\frac{T}{T_{\mathrm{VLT}}}\right)+2.075 .
\end{gathered}
$$

Here, the numerical coefficients are fitting parameters appli- cable to any collision and $T_{\mathrm{VLT}}$ is the only system-specific parameter which is calculated from the interaction parameters, see Eq. (9). The 1ist of $T_{\mathrm{VLT}}$ for all systems studied here is presented in Table III. The accurate and $\mathrm{ACCl}$ rate coefficients as well as the quality of the approximation by the amplification factor $A(T)$ from Eq. (31) are illustrated in Fig. 6.

Suppression factor. The chosen form of the suppression factor is

$$
S(T)=\frac{k_{j=1}^{\mathrm{B}}}{k_{j=1}^{\mathrm{ACCl}}(T)}\left[1+\left(\frac{k_{j=1}^{\mathrm{B}}}{k_{j=1}^{\mathrm{ACCl}}(T)}\right)^{n}\right]^{-1 / n} .
$$

There are two system-specific parameters, $k_{j=1}^{\mathrm{B}}$ and $n$. First, we observe that the values of $k_{j=1}^{\mathrm{B}}$ found from the zerotemperature extrapolation of the numerically determined rate coefficients are quite close to the estimate $k_{j=1 \text {,pert }}^{\mathrm{B}}$, see last column of Table III (for all the collisions, $k_{j=1, \text { pert }}^{\mathrm{B}}$ is about $5 \%$ higher than $k_{j=1}^{\mathrm{B}}$ ). Therefore, within the accuracy of about $5 \%, k_{j=1}^{\mathrm{B}}$ can be regarded as a system-specific nonfitting parameter. As for $n$, it remains an empirical fitting parameter. The list of $k_{j=1}^{\mathrm{B}}, k_{j=1 \text {, pert }}^{\mathrm{B}}$, and $n$ for all system collisions studied here presented in Table III.

Figure 7 shows accurate and $\mathrm{ACCl}$ rate coefficients as well as the functions $S(T)$ for $\mathrm{H}_{2}+\mathrm{H}_{2}^{+}, \mathrm{H}_{2}+\mathrm{H}_{3}^{+}$, and $\mathrm{H}_{2}$ $+\mathrm{CH}_{3}^{+}$collisions over the VLT range up to temperatures where convergence with the $\mathrm{ACCl}$ limit is obtained. A shal- 


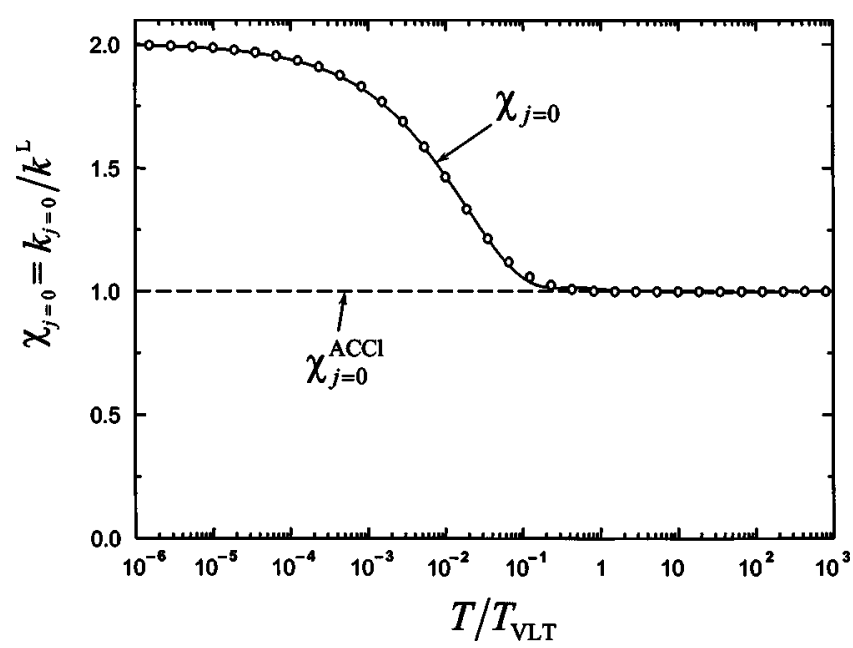

FIG. 6. Partner-independent accurate scaled total capture rate coefficients for $\mathrm{H}_{2}(j=0), \chi_{j=0}=k_{j=0} / k^{L}$, vs reduced temperature $T / T_{\mathrm{VLT}}$ across the VLT range, full line. The dashed line corresponds to the first-order $\mathrm{ACCl}$ approximation $\chi_{j=0}^{\mathrm{ACCl}}$ while the open circles provide the amplification factor $A$ from Eq. (31). Note that the scaling factor for the temperature $1 / T_{\mathrm{VLT}}$ is different for different collision partners.

low minimum in $k_{j=0}^{\mathrm{ACCl}}$ at the right-hand part of the figure, which is mentioned in Sec. III, is not discernable on the chosen scale of the vertical axis.

\section{CONCLUSION}

The rate coefficients for complex formation of homonuclear diatomic molecules in specified rotational states in collisions with ions are calculated within the $\mathrm{ACCl}$ approach for LT region and by the perturbative approximation at zero temperature (ZT, the Bethe limit). In the LT region, the di-

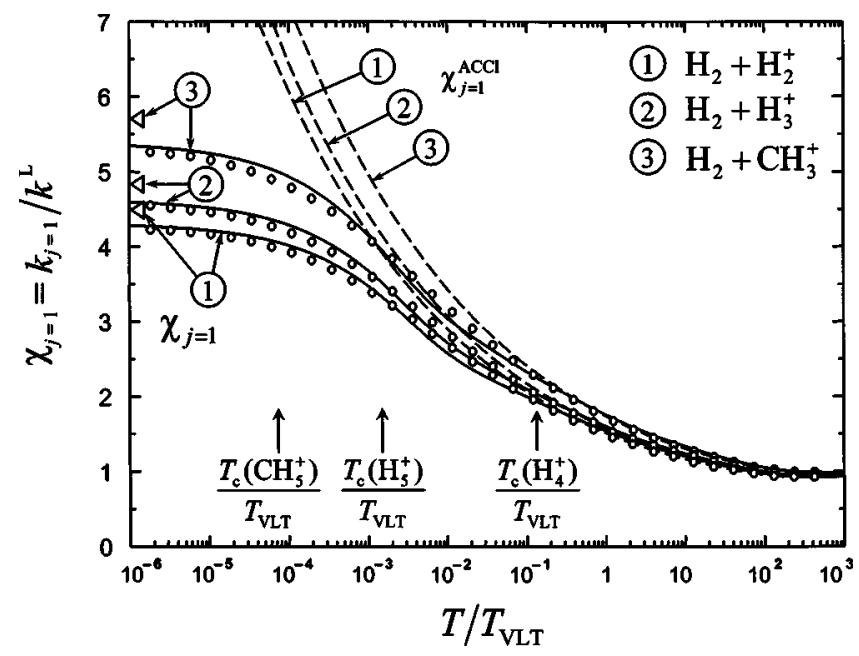

FIG. 7. Partner-specific accurate scaled total capture rate coefficients for $\mathrm{H}_{2}(j=1), \chi_{j=1}=k_{j=1} / k^{L}$, vs reduced temperature $T / T_{\mathrm{VLT}}$ across the VLT range. Results for $\mathrm{H}_{2}+\mathrm{H}_{2}^{+}, \mathrm{H}_{2}+\mathrm{H}_{3}^{+}$, and $\mathrm{H}_{2}+\mathrm{CH}_{3}^{+}$, collisions are shown by the full lines; triangles on the ordinate axis mark scaled zero-temperature limits of the capture rate coefficients $\chi_{j=1, \text { pert }}^{\mathrm{B}}=k_{j=1, \text { pert }}^{\mathrm{B}} / k^{L}$ [see Eq. (27)]. The dashed lines correspond to the $\mathrm{ACCl}$ approximation $\chi_{j=1}^{\mathrm{ACCl}}$ while the open circles correspond to the rate coefficients recovered from the suppression factor $S$ from Eq. (32). Note that the scaling factor for the temperature $1 / T_{\mathrm{VLT}}$ is different for different collision partners. The vertical arrows indicate the limits below which the statistical description of the capture may not be valid. mensionless rate coefficients $\chi_{j}^{\mathrm{ACCl}, \pm}$ for the even and odd cases (superscripts \pm ), calculated in the first-order PR approximation, are universal functions of the reduced temperature $T / T_{\mathrm{LT}}$ with $T_{\mathrm{LT}}=Q^{4} / q^{2} \alpha^{3} k_{B}$. In the second-order PR approximation, $\chi_{j}^{\mathrm{ACCl}, \pm}$ contain one diatom-specific parameter $\varepsilon_{0}=Q^{4} / q^{2} \alpha^{3} B$. The second-order PR approximation provides reliable values of the rate coefficients up to the rotational temperature $T_{\mathrm{rot}}=B / k_{\mathrm{B}}$. The ZT analytical expression for the capture rate constant $\chi_{j}^{\mathrm{B}, \pm}$ contains yet another parameter $\beta=\mu Q^{2} / \alpha \hbar^{2}$ which depends on the collision partner. In the region of VLT, which bridges the gap between the LT region and the ZT limit, the capture rate coefficients should be calculated from the solution of the coupled wave equations, or, within a good approximation, from the solution of uncoupled wave equations in an axially nonadiabatic basis. This is exemplified by the capture of hydrogen molecular isotopes molecules in the ground and the first excited rotational states with some hydrogen- and deuterium-containing ions (see Table III). Simple analytical expressions of the rate coefficients are derived which are valid in the whole temperature range below the rotational temperature.

The rate coefficients for hydrogen-ion collisions can be used for calculating the low-temperatures rate constants of ortho-para conversion of hydrogen molecules in collisions with polyatomic hydrogen-containing ions, which proceeds through the formation of an intermediate complex:

$$
\begin{aligned}
\mathrm{H}_{2}(j & =1, i=1)+X^{+}\left(j^{+},\left\{n^{+}\right\}, i^{+}\right) \\
& \rightarrow\left[\mathrm{H}_{2} X\right]^{+} \rightarrow \mathrm{H}_{2}\left(j^{\prime}=0, i^{\prime}=0\right)+X^{+}\left(j^{\prime+},\left\{n^{\prime+}\right\}, i^{\prime+}\right) .
\end{aligned}
$$

Here $i$ is the total nuclear spin of $\mathrm{H}_{2}$ and $X^{+}$is the ionic collision partner. The state of $\mathrm{H}_{2}$ is completely defined by two quantum numbers $j$ and $i$, with the latter being redundant in view of the exclusion principle. The state of $X^{+}$is specified by a set of quantum numbers, namely, by the total angular momentum $j^{+}$, a manifold of other internal quantum numbers $\left\{n^{+}\right\}$and the label $\Gamma$ of one of the irreducible representations of the permutation group of identical particles spanned by the spin (or coordinate) wave functions. ${ }^{25}$ For protons in $X^{+}$(particles with spin 1/2), the permutation property is completely defined by the total spin of nuclei $i^{+}$, which explains the appearance of the quantum numbers $j^{+}$, $\left\{n^{+}\right\}, i^{+}$in Eq. (33). ${ }^{26}$ For low temperatures (with partners $X^{+}$ in thermal equilibrium), the reaction in Eq. (33) is a twochannel process with respect to transitions in $\mathrm{H}_{2}$, but is a multichannel process with respect to transitions in $X^{+}$. The number of final states in $X^{+}$, populated as a result of the energy release in the reactive rotational deactivation of $\mathrm{H}_{2}$, depends critically on the density of states in $X^{+}$. In the general case, the ortho-para conversion rate constant $k_{j=1 \rightarrow j=0}^{\text {ortho-para }}$ can be written as

$$
k_{j=1 \rightarrow j=0}^{\text {ortho-para }}=C_{j=1 \rightarrow j=0}^{\text {ortho-para }} k_{j=1}^{\text {capture }},
$$

where $k_{j=1}^{\text {capture }}$ refers to the capture rate coefficient of $\mathrm{H}_{2}(j$ $=1$ ) by a structureless ion and the dimensionless coefficient $C_{j=1 \rightarrow j=0}^{\text {ortho-para }}$ describes the decay of the complex which depend on the structure of the ion. Explicit calculation of $C_{j=1 \rightarrow j=0}^{\text {ortho-para }}$, which is outside of this study, represents an interesting problem, addressed for a particular case $X^{+}=\mathrm{H}^{+}$in Ref. 27. 


\section{ACKNOWLEDGMENT}

Financial support of this work by the Deutsche Forschungsgemeinschaft (Grant No. SFB 357 "Molekulare Mechanismen unimolekularer Prozesse") is gratefully acknowledged.

\section{APPENDIX: AN INTEGRAL EXPRESSION OF THE CLASSICAL CAPTURE RATE CONSTANT FOR AN ARBITRARY POTENTIAL}

When calculating the capture rate coefficient within the $\mathrm{AC}$ approximation, one has first to calculate the partial rate coefficients, each for a specific AC potential. The standard way to do this is to determine the capture cross section for a given potential first, and then perform thermal averaging over the collision velocities. For an arbitrary potential, the first step does not yield an analytical expression for the capture cross section as a function of velocity and the second step requires a numerical integration (averaging in velocities) of the numerical integrand (cross section as a function of the velocity). It would be useful to skip the first stage and to express directly the capture rate constant as an integral from an expression that contains only the potential. For any given potential, the integral can be calculated numerically momentarily. The following shows that it is indeed possible to derive a formula of this kind. We begin with the capture conditions

$$
\begin{aligned}
& \frac{L_{m}^{2}}{2 \mu R_{m}^{2}}+U_{m}=\frac{p^{2}}{2 \mu}, \\
& -\frac{L_{m}^{2}}{\mu R_{m}^{3}}+U_{m}^{\prime}=0,
\end{aligned}
$$

where $U_{m}=U\left(R_{m}\right)$ and $U_{m}^{\prime}=d U\left(R_{m}\right) / d R_{m}, L_{m}$ is the capture angular momentum, and $R_{m}$ is the coordinate of the barrier maximum. From Eq. (A1) we get

$$
\begin{aligned}
& L_{m}^{2}=\mu R_{m}^{3} U_{m}^{\prime}, \\
& p^{2} / \mu=\left(R_{m} U_{m}^{\prime}+2 U_{m}\right) .
\end{aligned}
$$

Equation (A2) is now used to provide a parametric dependence of the capture cross section $\sigma_{c}=\pi L_{m}^{2} / p^{2}$ and the collision energy $E$ on the variable $R_{m}$ :

$$
\begin{aligned}
& \sigma_{c}=\pi L_{m}^{2} / p^{2}=\sigma_{c}\left(R_{m}\right), \\
& E=p^{2} / 2 \mu=E\left(R_{m}\right) .
\end{aligned}
$$

In principle, Eqs. (A3) define the energy dependence of the capture cross section on the collision energy if the variable $R_{m}$ is eliminated. In practice, this is not possible to accomplish analytically except for potentials of simple forms (say, inverse power-law potentials). The capture rate coefficient is

$$
k_{c}(T)=\int_{0}^{\infty}(p / \mu) \sigma_{c}(p) F(p, T) d p,
$$

with the Boltzmann distribution function and

$$
F(p, T) d p=\sqrt{2 / \pi}\left(\mu k_{\mathrm{B}} T\right)^{-3 / 2} \exp \left(-p^{2} / 2 \mu k_{\mathrm{B}} T\right) p^{2} d p .
$$

Thus,

$$
\begin{aligned}
k_{c}(T) & =\sqrt{2 \pi}\left(\mu k_{\mathrm{B}} T\right)^{-3 / 2} \int_{0}^{\infty} L_{m}^{2} \exp \left(-p^{2} / 2 \mu k_{\mathrm{B}} T\right)(p / \mu) d p \\
& =\sqrt{\pi / 2}\left(\mu k_{\mathrm{B}} T\right)^{-3 / 2} \int_{0}^{\infty} L_{m}^{2} \exp \left(-p^{2} / 2 \mu k_{\mathrm{B}} T\right) d\left(p^{2} / \mu\right) .
\end{aligned}
$$

Passing from the variable $p$ to the variable $R_{m}$ with the help of Eq. (A2) we obtain

$$
\begin{aligned}
k_{c}(T)= & -\sqrt{\pi / 2 \mu}\left(k_{\mathrm{B}} T\right)^{-3 / 2} \int_{0}^{R_{m}^{*}} R_{m}^{3} U_{m}^{\prime} \\
& \times \exp \left[-\left(R_{m} U_{m}^{\prime}+2 U_{m}\right) / 2 k_{\mathrm{B}} T\right] \\
& \times\left(U_{m}^{\prime \prime} R_{m}+3 U_{m}^{\prime}\right) d R_{m},
\end{aligned}
$$

where $\quad U_{m}=U\left(R_{m}\right), \quad U_{m}^{\prime}=d U\left(R_{m}\right) / d R_{m}, \quad$ and $\quad U_{m}^{\prime \prime}$ $=d^{2} U\left(R_{m}\right) / d R_{m}^{2}$. The upper integration limit in Eq. (A7), $R_{m}^{*}$ corresponds to the distance at which the potential $U(R)$ attains its maximum [if the potential $U(R)$ is repulsive at large distances and attractive at smaller distances]. In the case of pure attraction, $R_{m}^{*}=\infty$. The use of the integration variable $R_{m}$ is convenient since it allows one to easily monitor the important integration range and, therefore, to judge to what extent the neglect of a short-range part of the potential is meaningful.

Sometimes, it is convenient to replace the upper integration limit in Eq. (A7) by infinity making use of the Heaviside step function $\Theta$. Then Eq. (A7) assumes the form

$$
\begin{aligned}
k_{c}(T)= & -\sqrt{\pi / 2 \mu}\left(k_{\mathrm{B}} T\right)^{-3 / 2} \\
& \times \int_{0}^{\infty} R_{m}^{3} U_{m}^{\prime}\left(U_{m}^{\prime \prime} R_{m}+3 U_{m}^{\prime}\right) \Theta\left(U_{m}^{\prime}\right) \\
& \times \exp \left[-\left(R_{m} U_{m}^{\prime}+2 U_{m}\right) / 2 k_{\mathrm{B}} T\right] d R_{m} .
\end{aligned}
$$

This is the final formula which expresses the capture rate coefficient directly through the arbitrary interaction potential without intermediate determination of the capture cross section and which is convenient for numerical calculations.

${ }^{1}$ M. Quack and J. Troe, in Encyclopedia of Computational Chemistry, edited by P. V. Schleyer, N. L. Allinger, T. Clark, J. Gasteiger, and P. A. Kollmann (Wiley, Chichester, UK, 1998), Vol. 4, p. 2708.

${ }^{2}$ J. Troe, Adv. Chem. Phys. 82, 485 (1992).

${ }^{3}$ E. I. Dashevskaya, I. Litvin, E. E. Nikitin, and J. Troe, J. Chem. Phys. 120, 9989 (2004).

${ }^{4}$ E. I. Dashevskaya, I. Litvin, E. E. Nikitin, I. Oref, and J. Troe, J. Phys. Chem. A 108, 8703 (2004).

${ }^{5}$ D. Gerlich, in Book of Abstracts XIV Symposium in Atomic, Cluster and Surface Physics, Influence of the Exchange Symmetry on Low Temperature Ion-Molecule Reactions, edited by P. Casavechia (Aosta, Italy, 2004), pp. $1-4$.

${ }^{6}$ D. Gerlich and S. Schlemmer, Planet. Space Sci. 50, 1287 (2002).

${ }^{7}$ P. L. Chapovsky and L. J. F. Hermans, Annu. Rev. Phys. Chem. 50, 315 (1999).

${ }^{8}$ S. C. Smith and J. Troe, J. Chem. Phys. 97, 5451 (1992).

${ }^{9}$ A. I. Maergoiz and J. Troe, J. Chem. Phys. 99, 3218 (1993).

${ }^{10}$ A. I. Maergoiz, J. Troe, and Ch. Weiss, J. Chem. Phys. 101, 1885 (1994).

${ }^{11}$ A. I. Maergoiz, E. E. Nikitin, and J. Troe, Z. Phys. D: At., Mol. Clusters 
36, 339 (1996).

${ }^{12}$ A. I. Maergoiz, E. E. Nikitin, J. Troe, and V. G. Ushakov, J. Chem. Phys 105, 6270 (1996).

${ }^{13}$ H. A. Bethe, Rev. Mod. Phys. 9, 69 (1937).

${ }^{14}$ W. H. Miller, J. Chem. Phys. 52, 543 (1970).

${ }^{15}$ M. Berblinger, Ch. Schlier, J. Tennyson, and S. Miller, J. Chem. Phys. 96, 6842 (1992).

${ }^{16}$ J. Troe, Chem. Phys. 190, 381 (1995).

${ }^{17}$ V. Mandelshtam and H. S. Taylor, J. Chem. Soc., Faraday Trans. 93, 847 (1997).

${ }^{18}$ I. I. Fabrikant and H. Hotop, Phys. Rev. A 63, 022706 (2001).

${ }^{19}$ E. E. Nikitin, Theory of Elementary Atomic and Molecular Processes in Gases (Clarendon, Oxford, 1974).
${ }^{20}$ L. D. Landau and E. M. Lifshitz, Quantum Mechanics (Pergamon, Oxford, 1977).

${ }^{21}$ C. Eltschka, M. J. Moritz, and H. Friedrich, J. Phys. B 33, 4033 (2000). ${ }^{22}$ E. Vogt and G. H. Wannier, Phys. Rev. 95, 1190 (1954).

${ }^{23}$ E. I. Dashevskaya, I. Litvin, A. I. Maergoiz, E. E. Nikitin, and J. Troe, J. Chem. Phys. 118, 7313 (2003).

${ }^{24}$ D. A. Varshalovich, A. N. Moskalev, and V. K. Khersonskii, Quantum Theory of Angular Momentum (World Scientific, Singapore, 1988).

${ }^{25}$ M. Quack, Mol. Phys. 34, 477 (1977).

${ }^{26}$ R. Pauncz, The Construction of Spin Eigenfunctions: An Exercise Book (Kluwer Academic, New York, 2000).

${ }^{27}$ D. Gerlich, J. Chem. Phys. 92, 2377 (1990). 